\title{
Isolation of Methicillin Resistant - Staphylococcus aureus in Midwifery Students Faculty of Medicine Universitas Padjadjaran September-December 2012
}

\author{
Robinee Panirchelvam, ${ }^{1}$ Imam Megantara, ${ }^{2}$ Melati Sudiro ${ }^{3}$ \\ ${ }^{1}$ Faculty of Medicine Universitas Padjadjaran, ${ }^{2}$ Department of Microbiology Faculty of Medicine \\ Universitas Padjadjaran ${ }^{3}$ Department of Otorhinolaryngology, Head and Neck Surgery Faculty of \\ Medicine Universitas Padjadjaran/Dr. Hasan Sadikin General Hospital Bandung
}

\begin{abstract}
Background: Methicillin Resistant-Staphylococcus aureus (MRSA) is resistant bacteria to certain types of beta-lactam antibiotics. Commonly, the MRSA infection is acquired in hospitals, long-term care facilities, or similar institutional settings. Mid-wives are one of the health professional that have a major risk to get MRSA infection and could lead the infection among patients. This research is aimed to identify MRSA among the midwifery students

Methods: A descriptive study was conducted from September-December 2012 at the Microbiology laboratory to identify the proportion of MRSA among the mid-wife students from Faculty of Medicine, Universitas Padjadjaran. Twenty-five samples were chosen using simple random sampling based on their registration number. The inclusion criterias were healthy students, and 18 to 22 years old. Both nostrils were sampled with a sterile culture swab on both the subject's nostrils. Gram staining was done in order to identify the gram positive bacteria, Staphylococcus aureus. The samples were inaculated in Mannitol salt agar (MSA), incubated for 24 hours and at $37^{\circ} \mathrm{C}$. After 24 hours, catalase and coagulase tests were done. Moreover, for the susceptibility testing, the chosen media for this study was Mueller Hinton Agar (MHA) and with $30 \mu \mathrm{g}$ Cefoxitin disk. After incubation, the zone of inhibition of the colony less than $\leq 21 \mathrm{~mm}$ was considered resistant to methicillin.

Results: Colonization of MRSA was about 3 out from 25 samples

Conclusions: The MRSA colonization in the anterior nasal does exist in midwife students, a further study with more samples should be conducted. [AM].2015;2(1):204-7]
\end{abstract}

Key words: Midwife students, MRSA, Nasal Swab

\section{Introduction}

People around the world are suffering from various types of infections mainly caused by many types of bacteria. There are many treatments according to the type of causative agent. The main treatment of choice to treat bacterial infections is antibiotics. Some bacteria are resistant to the available antibiotics and their infections are difficult to be treated. Methicillin Resistant Staphylococcus aureus (MRSA), causes a serious health problem.1

MRSA is resistant to certain types of betalactam antibiotics, such as methicillin and other antibiotics such as oxacillin, penicillin, and amoxicillin. The infection from MRSA is mainly manifested by skin infection.2 Commonly, the infection is acquired in hospitals, longterm care facilities, or similar institutional settings. Prior to antibiotic exposure, a long stay in hospital, treated in the intensive care unit, underwent surgery before and most importantly, the exposure from MRSA infected patients will be the predisposing factors to be infected by MRSA.2 Disruption of normal skin structure and abnormal immunity due to improper hygiene and altered neutrophil function can make us to be more vulnerable to the infection..

In Indonesia, a population-based survey was conducted recently by the Antimicrobial Resistance in Indonesia, Prevalence and Prevention (AMRIN) Study Group in order to quantify human carrier of resistant microorganisms. A total of 3,995 individuals were screened between July and October 2001 in Surabaya and between January and May 2002 in Semarang, the rate of nasal carriage of Staphylococcus aureus was 362 out of

Correspondence: Robinee Panirchelvam, Faculty of Medicine, Universitas Padjadjaran, Jalan Raya Bandung-Sumedang Km.21, Jatinangor, Sumedang, Indonesia, Phone: +6287827793465 Fax: +6055279369 Email: robineepanir@yahoo.com 
3,995 (9.1\%) individuals and about 90/361 (24.9\%) Staphylococcus aureus strains were resistant to tetracycline and $2 / 361(0.6 \%)$ were resistant to oxacillin.3 The prevalence of MRSA in Atmajaya Hospital , Jakarta in the year 2003 was $47 \% .4$ In Bandung, a study had been done on the lecturers of Medical Faculty of Universitas Padjadjaran and the result was about $52.9 \%$ out of 20 samples were MRSA.5 Another was also done on the students in Medical Faculty of Universitas Padjadjaran and the result was about $14.29 \%$ MRSA found among 70 samples taken. 6

Colonization of MRSA is usually on the anterior nasal. It can resides in the nasal without causing any illnesses to people who carrying the MRSA.7 This people can act as carriers that can readily transmit MRSA to other people. This situation may be harmful if they are transmitted to patients who are very ill or immuno-incompetent. 2 When they are infected, there are possibilities that these patients will face some serious complications. 8

In Indonesia, the mid-wives are usually sent to the rural area to work and the community from the lower socio-economy usually seeks their service because it is much cheaper to get the services from a mid-wife compared to the service from a medical doctor. Most importantly, the mid-wife students are those without the exposure of MRSA from health care settings, so it will be purely from the community based with and without health care exposure. So, a study has to be done in order to find out do MRSA really exist among these students before their working career.

The data for MRSA prevalence in Indonesia is still poor9; studies must be conducted to know the prevalence and the emerging of the MRSA infection in Indonesia. By knowing the prevalence of the MRSA, we can prevent the MRSA associated infections by conducting public health programs to provide awareness to the medical personnel and to the public on the seriousness of this infection. This is to ensure a healthier living and a promising future for the community. Moreover, the cost to treat MRSA infection is very expensive. So, the lower socio-economic income people will be stigmatized to get the proper medication and cure. So in conclusion, prevention is always better than cure.

\section{Methods}

A descriptive study was conducted from September 2012 - December 2012 at the
Microbiology laboratory to identify the proportion frequency of MRSA among the mid-wife students from Faculty of Medicine, Universitas Padjadjaran. 25 samples were choosen using a simple random sampling based on their registration numbers.

The inclusion criteria were students who were still doing the diploma program in midwifery in Faculty of Medicine, Universitas Padjadjaran and healthy during the sample collection, all female and around the age of 18 to 22 years old. Those who did not fill up the informed consent form, ill (having flu, sinusitis), having prior antibiotic exposure before and during sample collection and failed to present on the day of sample collection were excluded from the study to avoid bias.

Both nostrils were sampled with a sterile culture swab on both the subject's nostrils.10 After getting the samples from the anterior nares, gram staining was done in order to identify the gram positive bacteria, Staphylococcus aureus. Gram positive bacteria retained the crystal violet stain, while the gram negative bacteria lose the crystal violet stain and stained by the safranin and appear red.

The chosen medium for growth for this MRSA culture was Mannitol salt agar (MSA). After the inoculation, the agar was incubated for 24 hours and at $37^{\circ} \mathrm{C}$. After 24 hours, the colonies were identified by the appearance of the color of the colony. The convex colonies with light to golden yellow colonies were assumed as Staphylococcus aureus. 11

The catalase test was used to identify the gram positive cocci and some gram positive bacilli. So after gram staining, catalase was done to differentiate streptococcus and staphylococcus. When bubbles appeared, then it was recorded as positive catalase. 12

A coagulase test was done to differentiate Staphylococcus aureus with Staphylococcus epidermidis. Clumping of cocci within 5-10 seconds was taken as positive for Staphylococcus aureus. Some strains of Staphylococcus aureus may not produce a bound coagulase, and such strains were identified by tube coagulase test.13 Positive result was indicated by gelling of the plasma, which remained in place even after inverting the tube.

For the susceptibility testing, the chosen media for this study was Mueller Hinton Agar (MHA) and with 30 $\mu$ g Cefoxitin disk14 because recent reports had shown that Cefoxitin was a better agent for prediction of MRSA15. With disc diffusion method, each quadrant was placed with a $30 \mu \mathrm{g}$ of Cefoxitin 
discs and incubated at $33-35^{\circ} \mathrm{C}$ at ambient air for 16 to 18 hours.

After incubation the zone of inhibition of the colony less than $\leq 21 \mathrm{~mm}$ was considered resistant to methicillin. The others were considered as negative.

\section{Results}

Staphylococcus aureus were colonized in the anterior nasal of midwife students in the study and were proven in 11 samples with gram staining which showed gram positive diplococci in clusters under the microscope, growth of yellow colonies in the Mannitol Salt Agar, positive catalase test and positive coagulase test done on the yellow colony. From 11 samples proven, 3 were MRSA positive.

\section{Discussions}

It can be clearly seen that colonization of MRSA is still low among the midwife students because the frequency of MRSA in this study is 3 out from 25 samples. The fact that MRSA exists in the community is proven through this research.

The result in this study similar with a study by Rianti,6 who stated that frequency was $14.29 \%$. This study concludes and supports the fact that community in Faculty of Medicine, Universitas Padjadjaran are exposed to MRSA colonization in the anterior nasal. It gives us the clue indirectly that, communities in Jatinangor are exposed to MRSA too. More studies should be done to obtain data on prevalence of MRSA, in order to prevent unwanted complications due to MRSA infections.

In contrast, in Indonesia, a research done by Antimicrobial Resistance in Indonesia, Prevalence and Prevention (AMRIN) Study Group in Semarang, revealed that $0.6 \%$ were resistant to oxacillin in 2002 9. This could be the cause this research was done in the year 2002;. There is a huge gap of time, and maybe the MRSA would have increased by now. So, more researches must be conducted to obtain current and updated frequency of MRSA in the community of Indonesia.

Besides that, the study which was conducted by Nordin 5 in Faculty of Medicine, Universitas Padjadjaran, showed that the prevalence was $52.9 \% 5$, this is contrary to the result obtained in this study and maybe it is due to the reason that the lecturers are exposed to hospital settings in daily basis. It could have influence the outcome due to the strong association of MRSA exposure in hospitals or health care centers 4.

There are several limitations that would have caused bias in this research. The time and cost factor is one of the limitations. In a much stipulated time and small fund, data collection is done from small scale of samples. Identification of frequency of MRSA among all the midwife students would have produced a better result. .

In summary, it is known that the MRSA colonization in the anterior nasal do exist in midwife students with 3 out of 25. Measures should be taken to further study on MRSA colonization among students who are going to pursue medical careers in order to prevent them from spreading it to the community in the future, both the health care settings and community based settings. Serious actions have to be taken to stop the cycle of MRSA exposure.

\section{References}

1. M'Ikanatha NM, Lynfield R, Van Beneden CA, de Valk H, Editors. Infectious disease surveillance. 1st ed. Oxford: WileyBlackwell Pub; 2007

2. Centers for Disease Control and Prevention. Methicillin-resistant Staphylococcus Aureus (MRSA) Infections. Atlanta; 2010 [updated April 15, 2011] [Cited 2012 April 25]. Available from: http://www.cdc. gov/mrsa/definition/index.html.

3. Crossley KB, Jefferson KK, Archer GL, Fowler VG. Staphylococci in Human Disease. 2nd ed. Oxford: Wiley-Blackwell; 2009. [Cited 2012 May 20]. Available from: http://onlinelibrary.wiley.com/ doi/10.1002/9781444308464.fmatter/ pdf

4. Noviana H. Isolasi dan uji kepekaan isolat klinis ORSA dan nonORSA terhadap vankomisin dan antibiotik lainnya. Jurnal Mikrobiologi Indonesia. 2004;9(2):51-4.

5. Nordin ARB. Detection of MRSA on nasal swab of lecturers in Faculty of Medicine, Universitas Padjadjaran [Minor Thesis]. Bandung: Universitas Padjadjaran; 2012.

6. Rianti N. Deteksi MRSA dari apus hidung mahasiswa FK UNPAD angkatan 2007 [Minor Thesis];Bandung: Universitas Padjadjaran; 2011.

7. Hanumanthappa AR, Chandrappa NR, Rajasekharappa MG. Prevalence of methicillin resistant Staphylococcus aureus in Karnataka. Indian J Pathol 
Microbiol. 2003;46(1):129-32.

8. Bevis M. MRSA evolves as it moves between humans and livestock. 2005 [Cited 2012 May 14]. Available from: http://knau.org/ post/mrsa-evolves-it-moves-betweenhumans-and-livestock.

9. Lestari ES, Severin JA, Filius PM, Kuntaman K, Duerink DO, Hadi U, et al. Antimicrobial resistance among commensal isolates of Escherichia coli and Staphylococcus aureus in the Indonesian population inside and outside hospitals. Eur J Clin Microbiol Infect Dis. 2008;27(1):45-51.

10. Standford Hospital \& Clinics Infection Control and Lucile Packard Children's Hospital. Nares culture for MRSA screening. 2009.[Cited 2012 June 17] Available from: https://lane.stanford.edu/portals/cvicu/ HCP_Misc._Tools/NARES_CULTURE_FOR_ MRSA_SCREENING.pdf.

11. Clinical and Laboratory Standards Institute. Performance standards for antimicrobial susceptibility testing; Twentieth Informational Supplement. CLSI document M100-S20. Wayne PA: Clinical and Laboratory Standard Institute; 2010

12. CDC. Laboratory Detection of Oxacillin/ Methicillin-resistant Staphylococcus aureus. Centres for Disease Control and prevention; 2010 [updated August 9, 2010] [Cited 2012 May 20]. Available from: http://www.cdc. gov/mrsa/lab/lab-detection.html.

13. Asbell PA, Sahm DF, Shaw M, Draghi DC, Brown NP. Increasing prevalence of methicillin resistance in serious ocular infections caused by Staphylococcus aureus in the United States: 2000 to 2005. J Cataract Refract Surg. 2008;34(5):814-8.

14. ChingChingWu. Disk Diffusion Susceptibility Testing (Kirby-Bauer Method). University West Lafayette. 2002 [Cited 2012 July 5]. Available from: http://www.addl.purdue.edu/ newsletters/1997/spring/dds.shtml.

15. Smyth RW, Kahlmeter G. Mannitol salt agar-cefoxitin combination as a screening medium for methicillinresistant Staphylococcus aureus. Clin Microbiol. 2005;43(8):3797-9. 